\title{
The influence of anesthesia- body mass index and chronicity of the injury on the reliability of diagnostic tests for anterior cruciate ligament rupture
}

\author{
Emre Bilgin',* , Ali Turgut², Sertan Hancıoğlu ${ }^{3}$, Emre Sarıekiz², Melikşah Uzakgider', Önder Kalenderer ${ }^{4}$ \\ 'Department of Orthopaedics and Traumatology, Bozyaka Training and Research Hospital, Health Science University, Izmir, Turkey \\ 2Department of Orthopaedics and Traumatology, Tepecik Training and Research Hospital, Health Science University, Izmir, Turkey \\ ${ }^{3}$ Department of Orthopaedics and Traumatology, Faculty of Medicine, Celal Bayar University, Manisa, Turkey \\ ${ }^{4}$ Department of Orthopaedics and Traumatology, Faculty of Medicine, Tinaztepe University, Izmir, Turkey
}

This study aimed to analyze the accuracy and interobserver reliability of the four common diagnostic tests for anterior cruciate ligament (ACL) rupture. The effect of anesthesia, chronicity of the injury and patient's body mass index (BMI) on the reliabilities was also assessed. Patients who underwent arthroscopic knee surgery were examined before the surgery and under anesthesia by three observers categorized based on their experience levels. One hundred two patients were evaluated to determine the accuracy of these tests. Sixty-two patients with ACL rupture were further examined to assess the effect of BMI $\left(\leq 25 \mathrm{~kg} / \mathrm{m}^{2}\right.$, or $>25 \mathrm{~kg} / \mathrm{m}^{2}$ ) and chronicity ( $\leq 4$ or $>4$ weeks) of the injury on reliabilities with using the Fleiss kappa method. The Lachman test performed under anesthesia had the highest sensitivity (100\%, 100\%, and 96.7\%). In contrast, pivot shift and lever sign tests had the lowest sensitivity both in awake $(24.2 \%, 17.7 \%, 8.1 \%$ and $37.1 \%, 33.8 \%, 29 \%)$ and anesthetized condition $(75.8 \%, 75.8 \%, 67.7 \%$ and $41.9 \%, 43.5 \%, 40.3 \%)$. Specificities of the tests except pivot shift were negatively affected when performed under anesthesia. The reliability of the anterior drawer test was perfect under anesthesia. The lever sign test had the highest reliability in the awake condition in patients with a BMI of $>25 \mathrm{~kg} / \mathrm{m}^{2}$. Furthermore, the reliabilities of the anterior drawer, Lachman, and pivot-shift tests in awake condition were found to be increased in patients with a chronic injury. Finally, the examiner's experience is not important for the physical diagnosis of ACL rupture.

Keywords: Anterior cruciate ligament, Diagnostic test, Accuracy, Interobserver reliability, Body mass index, Experience

\section{INTRODUCTION}

The anterior cruciate ligament $(\mathrm{ACL})$ is one of the most frequently injured ligaments among the knee joint. ACL rupture results in anterior instability of the knee, and this subsequently leads to cartilage or meniscal injury. Therefore early diagnosis and treatment is important to obtain optimal results. The diagnosis of ACL rupture can be made by physical examination, magnetic resonance imaging (MRI), and arthroscopic visualization (Jarbo et al., 2017; Lange et al., 2015).

Physical examination is the first step in the diagnosis. Anterior drawer, Lachman, and pivot-shift tests have been using more than
30 years to recognize the ACL rupture (Galway and Maclntosh, 1980; Marshall et al., 1975; Torg et al., 1976). Recently, Lelli et al. (2016) introduced the fourth frequently used clinical test: "the lever sign test." The reliability of these four tests is crucial in clinical practice. Thus, additional costs caused by unnecessary MRI can be lowered, excluding the presence of injury by accurate physical examination methods.

These tests' accuracy can be affected by patient factors such as; pain, muscle contraction, leg weight, etc. On the other hand, the examiner's experience is also an essential factor influencing a diagnostic test's accuracy. The accuracy of these tests was investigated widely previously (Abruscato et al., 2019; Deveci et al., 2015; Jar-
${ }^{*}$ Corresponding author: Emre Bilgin (iD https://orcid.org/0000-0003-3989-6732 Department of Orthopaedics and Traumatology, Bozyaka Training and Research Hospital, Health Science University, İzmir, Turkey Email: dremrebilgin@hotmail.com

Received: October 1, 2021 / Accepted: October 20, 2021
This is an Open Access article distributed under the terms of the Creative Commons Attribution Non-Commercial License (https://creativecommons.org/licenses/by-nc/4.0/) which permits unrestricted non-commercial use, distribution, and reproduction in any medium, provided the original work is properly cited. 
bo et al., 2017; Lichtenberg et al., 2018; Mulligan et al., 2017; Reiman et al., 2018). However, there is not enough data about the influence of patient-related factors on the accuracy and reliability of the diagnostic physical examination tests for ACL injury in the literature.

The purpose of this study was to investigate the accuracy and interobserver reliability of anterior drawer, Lachman, pivot shift, and lever sign tests, as well as the effect of patient factors such as; being under anesthesia and at the acute stage of the injury ( $\leq 4$ weeks), and with body mass index (BMI) equal or under $25 \mathrm{~kg} / \mathrm{m}^{2}$ on the reliability of these tests. Our hypotheses were that; the accuracy of the diagnostic physical examination tests for ACL injury would be higher if the patient is under anesthesia. Moreover, interobserver reliabilities should be higher if the injury is chronic and the patient's BMI is under or equal to $25 \mathrm{~kg} / \mathrm{m}^{2}$.

\section{MATERIALS AND METHODS}

The local ethical committee approved this prospectively designed diagnostic study (approval number: 2019/11-2, Health Science University Tepecik Training and Research Hospital). The study was conducted between February 2019 and December 2020. The patients who underwent arthroscopic knee surgery because of ACL or meniscal injury between the age of 17 and 40 years old were included in the study. Written consent was obtained from all the patients. Exclusion criteria were; patients who were older than the age of 40 years (because of possible joint degeneration), patients with previous knee surgery on the same or the other side, having previously suspicious ACL lesion on the opposite knee, and who will need a knee surgery other than meniscal or ACL surgery (high tibial osteotomy, multi-ligamentous injury, etc.). Besides, patients with a locked knee in preoperative examination and patients who had had meniscal root tear based on MRI (because of its effect on anteroposterior stability of the knee) and partial ruptures of ACL were excluded after the arthroscopic examination.

Initially, based on the criteria above, 65 patients with ACL rupture and 42 patients with intact ACL based on MRI were included. After the arthroscopic visualization, three patients in the ruptured ACL group and two patients in the intact ACL group had a partial rupture; therefore they were excluded. Overall, data of 102 patients either with complete ruptured ACL $(n=62)$ or intact ACL ( $\mathrm{n}=40$ ) were included in the study.

The first author collected the patients' data (age, gender, side and time of the injury, and MRI findings). Three observers who were blinded to the MRI and previous physical examination find- ings were asked to examine the patients one day before the surgery at the clinic and after induction of the anesthesia at the operation room. The observers were selected in three experience level groups as; a resident, a junior surgeon, and a senior surgeon involved in the study group after collecting the data. A brief lecture about detailed information on physical examination methods was given to the observers at the beginning of the study.

\section{Anterior drawer test}

The patient lies in a supine position. The hip and knee are flexed to $45^{\circ}$ and $90^{\circ}$, respectively. The fleet is flat on the table. The examiner's thumbs are positioned on both medial and lateral sides of the patellar tendon, and the other four fingers grasp the proximal tibia. The proximal tibia is pulled anteriorly. An increased amount of anterior tibial translation than the healthy side is accepted as a positive finding as an ACL injury (Marshall et al., 1975).

\section{Lachman test}

The patient lies in a supine position. The knee is flexed to $15^{\circ}-$ $30^{\circ}$, and the examiner grasps the patient's distal thigh with one hand and grasps the proximal tibia with the other hand. The thumb of the hand which holds the proximal tibia should be on the tibial tuberosity. The tibia is pulled anteriorly, and the anterior motion of the tibia compared to the femur is noted. The opposite side can be evaluated if it is thought to have an intact ACL for comparison. The observers were asked to decide whether the ACL is intact or not (Torg et al., 1976).

\section{Pivot-shift test}

The patient lies in a supine position. The hip is passively flexed to $40^{\circ}$ and abducted to relax the iliotibial band. The examiner stands on the lateral side of the affected knee. The lower leg-ankle is grasped with maintaining internal rotation. The knee is allowed to be in full extension. The other hand holds the leg's lateral side at the proximal tibiofibular joint level with increasing the internal rotation. While the knee is passively flexed, valgus and axial force is applied to the knee. If the knee is subluxated due to ACL deficiency at the extension position, it spontaneously reduces at the range of $30^{\circ}$ to $40^{\circ}$ flexion by this maneuver (Lichtenberg et al., 2018).

\section{Lever sign test}

The patient lies in a supine position with the knees fully extended. The examiner stands at the injured knee's side and puts a closed fist under the proximal $1 / 3$ of the calf, which causes a slight 
flexion at the knee joint. Afterward, the examiner applies a downward force to the distal $1 / 3$ of the quadriceps. If the ACL is deficient, the heel will not rise off the examination table (Lelli et al., 2016). An arthroscopic finding of the continuity of ACL was accepted as an exact diagnosis. The specificity, sensitivity, positive predictive value, and negative predictive value were calculated with the observers' findings and the reference finding of arthroscopic examination. After evaluating the tests' accuracy, a further investigation was performed, including the patients' data with a complete rupture of ACL. These patients were divided into two groups based on the period between the injury and evaluation as; acute ( $\leq 4$ weeks since injury) or chronic (Jarbo et al., 2017), based on the BMI of the patient as; BMI value of $\leq 25$ or $>25 \mathrm{~kg} / \mathrm{m}^{2}$. Thirty-three of the patients (53.2\%) had an acute injury, 41 (66.1\%) of the patients' BMI value was $\leq 25 \mathrm{~kg} / \mathrm{m}^{2}$.

\section{Statistical analysis}

Statistical analysis was performed using IBM SPSS Statistics ver. 24.0 (IBM Co., Armonk, NY, USA). Demographic characteristics of the patients were given as mean \pm standard deviation. The interobserver reliabilities were calculated with a Fleiss kappa method. A value $>0.80$ was interpreted as almost perfect agreement, 0.61 to 0.80 as substantial agreement, 0.41 to 0.60 as moderate agreement, 0.21 to 0.40 as fair agreement, and $<0.21$ as slight or poor agreement (Lichtenberg et al., 2018).

\section{RESULTS}

Of all patients, 21 (20.5\%) were female. The right knee was injured in 68 patients $(66.6 \%)$. The mean age of the patients was $27.2 \pm 6.8$ years (range, $17-40$ years). The demographic characteristics of the patients are given in Table 1.

It was observed that the sensitivity of the Lachman test, which was performed under anesthesia, was the highest; in contrast, pivot shift and lever sign tests had the lowest sensitivities both when performed one day before the surgery and under anesthesia (Table 2). The level of experience was not found to be important about the sensitivity of the tests. Sensitivities were positively affected by performing the tests under anesthesia (Table 2). Specificities were found to be better than sensitivities for all tests. Besides, it was observed that the tests' specificities were affected adversely when they were

Table 1. The demographic characteristics of the patients

\begin{tabular}{lccc} 
Characteristic & $\begin{array}{c}\text { ACL rupture group } \\
(\mathrm{n}=62)\end{array}$ & $\begin{array}{c}\text { ACL intact group } \\
(\mathrm{n}=40)\end{array}$ & $\begin{array}{c}\text { Total } \\
(\mathrm{n}=102)\end{array}$ \\
\hline Gender, female:male & $5: 57$ & $16: 24$ & $21: 81$ \\
Operative side, right:left & $42: 20$ & $26: 14$ & $68: 34$ \\
Age $(\mathrm{yr})$ & $24.6 \pm 5.1$ & $31.2 \pm 7.5$ & $27.2 \pm 6.8$ \\
$\mathrm{BMI}\left(\mathrm{kg} / \mathrm{m}^{2}\right)$ & $24.0 \pm 2.5$ & $26.9 \pm 3.9$ & $25.2 \pm 3.4$ \\
\hline
\end{tabular}

Values are presented as number or mean \pm standard deviation.

$\mathrm{ACL}$, anterior cruciate ligament; $\mathrm{BMI}$, body mass index.

Table 2. Specificity, sensitivity, positive predictive, and negative predictive values of physical examination tests for ACL rupture both awake and under anesthesia among different experience levels

\begin{tabular}{|c|c|c|c|c|c|c|c|c|}
\hline Variable & ADT & ADTA & LT & LTA & PST & PSTA & LST & LSTA \\
\hline \multicolumn{9}{|l|}{ Sensitivity (\%) } \\
\hline Senior surgeon & 90.3 & 98.3 & 96.7 & 100 & 24.2 & 75.8 & 37.1 & 41.9 \\
\hline Specialist & 90.3 & 98.3 & 88.7 & 100 & 17.7 & 75.8 & 33.8 & 43.5 \\
\hline Resident & 85.4 & 98.3 & 87.1 & 96.7 & 8.1 & 67.7 & 29.0 & 40.3 \\
\hline \multicolumn{9}{|l|}{ Specificity (\%) } \\
\hline Senior surgeon & 92.5 & 87.5 & 100 & 100 & 100 & 100 & 100 & 97.5 \\
\hline Specialist & 95.0 & 75.0 & 100 & 97.5 & 100 & 100 & 100 & 97.5 \\
\hline Resident & 85.0 & 80.0 & 100 & 92.5 & 100 & 100 & 100 & 97.5 \\
\hline \multicolumn{9}{|c|}{ Positive predictive value (\%) } \\
\hline Senior surgeon & 94.9 & 92.4 & 100 & 100 & 100 & 100 & 100 & 96.3 \\
\hline Specialist & 96.5 & 85.9 & 100 & 98.4 & 100 & 100 & 100 & 96.4 \\
\hline Resident & 89.8 & 88.4 & 100 & 95.2 & 100 & 100 & 100 & 96.1 \\
\hline \multicolumn{9}{|c|}{ Negative predictive value (\%) } \\
\hline Senior surgeon & 90.2 & 97.2 & 95.2 & 100 & 45.9 & 72.7 & 50.6 & 52.0 \\
\hline Specialist & 86.3 & 96.7 & 85.1 & 100 & 43.9 & 72.7 & 49.3 & 52.7 \\
\hline Resident & 79.0 & 96.9 & 83.3 & 94.8 & 41.2 & 66.6 & 47.6 & 51.3 \\
\hline
\end{tabular}

ACL, anterior cruciate ligament; ADT, anterior drawer test; ADTA, anterior drawer test under anesthesia; LT, Lachman test; LTA, Lachman test under anesthesia; PST, pivot-shift test; PSTA, pivot-shift test under anesthesia; LST, lever sign test; LSTA, lever sign test under anesthesia. 
Table 3. Interobserver reliabilities of the physical examination tests for ACL rupture in acute and chronic trauma for all patients

\begin{tabular}{lccc}
\hline Physical examination tests & Overall & Acute injuries $(\mathrm{n}=33)$ & Chronic injuries $(\mathrm{n}=29)$ \\
\hline ADT & $0.648(0.505-0.792)$ & $0.591(0.394-0.788)$ & $0.725(0.515-0.935)$ \\
ADTA & $1.000(0.856-1.144)$ & $1.000(0.803-1.197)$ & $1.000(0.797-1.198)$ \\
LT & $0.552(0.408-0.696)$ & $0.511(0.314-0.708)$ & $0.584(0.374-0.794)$ \\
LTA & $-0.011(-0.155$ to 0.133$)$ & $-0.010(-0.207$ to 0.187$)$ & $-0.012(-0.222$ to 0.199$)$ \\
PST & $0.574(0.430-0.718)$ & $0.489(0.292-0.686)$ & $0.610(0.400-0.821)$ \\
PSTA & $0.863(0.720-1.007)$ & $0.847(0.650-1.044)$ & $0.882(0.672-1.092)$ \\
LST & $0.879(0.735-1.023)$ & $0.916(0.719-1.113)$ & $0.817(0.607-1.028)$ \\
LSTA & $0.956(0.812-1.100)$ & $1.000(0.803-1.197)$ & $0.902(0.692-1.112)$
\end{tabular}

Values are presented as Fleiss kappa (95\% confidence interval).

ACL, anterior cruciate ligament; ADT, anterior drawer test; ADTA, anterior drawer test under anesthesia; LT, Lachman test; LTA, Lachman test under anesthesia; PST, pivot-shift test; PSTA, pivot-shift test under anesthesia; LST, lever sign test; LSTA, lever sign test under anesthesia.

Table 4. Interobserver reliabilities of the physical examination tests for ACL rupture based on body mass index (BMI) values of the patients

\begin{tabular}{lcc}
\hline Physical examination tests & $\mathrm{BMl} \leq 25 \mathrm{~kg} / \mathrm{m}^{2}(\mathrm{n}=41)$ & $\mathrm{BMl}>25 \mathrm{~kg} / \mathrm{m}^{2}(\mathrm{n}=21)$ \\
\hline ADT & $0.678(0.501-0.854)$ & $0.566(0.319-0.812)$ \\
ADTA & $1.000(0.767-1.779)$ & $1.000(0.753-1.247)$ \\
LT & $0.641(0.464-0.817)$ & $0.199(-0.048-0.446)$ \\
LTA & $-0.008(-0.185$ to 0.169$)$ & $-0.016(-0.263$ to 0.231$)$ \\
PST & $0.544(0.368-0.721)$ & $0.612(0.365-0.859)$ \\
PSTA & $0.876(0.699-1.052)$ & $0.839(0.592-1.086)$ \\
LST & $0.855(0.679-1.032)$ & $0.927(0.680-1.174)$ \\
LSTA & $0.934(0.757-1.111)$ & $1.000(0.753-1.247)$
\end{tabular}

Values are presented as Fleiss kappa (95\% confidence interval).

$\mathrm{ACL}$, anterior cruciate ligament; $\mathrm{ADT}$, anterior drawer test; ADTA, anterior drawer test under anesthesia; LT, Lachman test; LTA, Lachman test under anesthesia; PST, pivot-shift test; PSTA, pivot-shift test under anesthesia; LST, lever sign test; LSTA, lever sign test under anesthesia.

performed under anesthesia (except pivot-shift test). The specificity of anterior drawer tests was the lowest (Table 2). Similarly, the experience did affect the specificity levels of the tests.

Overall, the anterior drawer test performed under anesthesia had the highest interobserver reliability (Fleiss kappa:1) (Table 3). The injury was acute in 33 patients $(53.2 \%)$. The interobserver reliabilities of the anterior drawer, Lachman, and pivot shift (both awake and under anesthesia) tests were found to be increased in patients with a chronic injury (Table 3).

Forty-one of the patients $(66.1 \%)$ were with a BMI value of $\leq 25$ years. The interobserver reliability of anterior drawer, Lachman (both awake and under anesthesia), and pivot shift (under anesthesia) tests were found to be decreased in patients with a BMI value of $>25$ years. In contrast, the interobserver reliability of the lever sign test (both awake and under anesthesia) was found to be increased in patients with a BMI value of $>25$ years (Table 4 ). The kappa values of the patients among the groups are given as a graphic in Fig. 1.

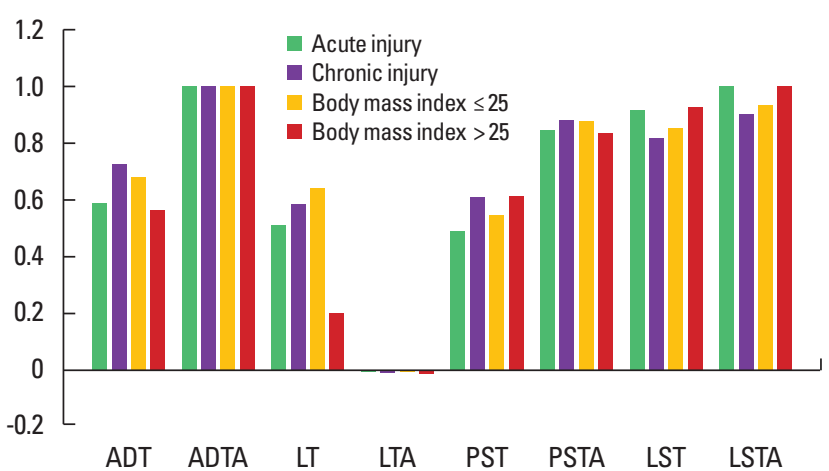

Fig. 1. Graphic for the reliabilities of the physical examination tests in different scenarios. ADT, anterior drawer test; ADTA, anterior drawer test under anesthesia; LT, Lachman test; LTA, Lachman test under anesthesia; PST, pivot-shift test; PSTA, pivot-shift test under anesthesia; LST, lever sign test; LSTA, lever sign test under anesthesia.

\section{DISCUSSION}

ACL injuries are common in orthopedic practice. An accurate physical examination method is an indispensable step in diagnosing ACL lesions as in any disease. In this study, we aimed to compare the accuracy of frequently used physical examination tests for ACL injury in awake and anesthetized patients. Additionally, the interobserver reliabilities were evaluated based on some scenarios such as the time after injury, the rate of the patient's BMI, and the state of being under anesthesia.

Our study showed that; sensitivities of all four tests were higher when performed under anesthesia. In contrast, specificities of the tests were affected negatively when they were performed under anesthesia. The lowest sensitivity was observed in the pivot-shift test in the patients without anesthesia. However, under anesthesia, the lowest sensitivity was observed in the lever sign test. The specificity of the anterior drawer test was the lowest when performed 
on both awake and anesthetized patients. The interobserver reliability of anterior drawer and pivot shift (both awake and under anesthesia) and Lachman (awake) tests were found to be increased in patients with a chronic injury. Only the lever sign test's interobserver reliability was not affected negatively in patients with a BMI of $>25 \mathrm{~kg} / \mathrm{m}^{2}$.

The Lachman test has been accepted as the most sensitive test for evaluating the ACL injury (Jarbo et al., 2017; Logan et al., 2004). The sensitivity of the pivot shift test is generally considered the lowest (Jarbo et al., 2017; Scholten et al., 2003). The lever sign test is the most recently developed one (Lelli et al., 2016). Lelli et al. (2016) and Deveci et al. (2015) stated that the most sensitive test was the lever sign test. However, the results of Jarbo et al. (2017) and Mulligan et al. (2017) did not confirm this high sensitivity, neither do our findings.

Pain sensation and reflex muscular contraction are the main issues that can affect diagnostic tests' accuracy when the patient is awake or under anesthesia. One can expect that the pain sensation would not obscure the diagnostic test's success if the patient is under anesthesia. Jarbo et al. (2017) evaluated their patients both awake and under anesthesia using MRI findings to confirm the diagnosis. They calculated the accuracy by averaging sensitivity and specificity percentages. The conclusion was that; the most accurate test was Lachman in awake patients (90\%) when it was the anterior drawer and the Lachman tests in anesthetized patients (96\% for both). The least accurate tests were pivot shift in awake patients and lever sign in anesthetized patients $(65 \%$ and $76 \%$, respectively). Deveci et al. (2015) similarly evaluated the four tests' sensitivity and specificities in awake and anesthetized 117 patients. According to their results, the lowest and highest sensitivity in awake patients were in anterior drawer and lever sign tests, respectively (60\% and 94\%, respectively). In anesthetized patients, sensitivities of Lachman, anterior drawer, and pivot-shift tests were the same (88\%), and the lever sign test's sensitivity was much higher (98\%). In our study, the lowest and highest sensitivity in awake patients were observed in pivot-shift and Lachman tests, respectively (meanly $16.7 \%$ and $90.8 \%$, respectively). The lowest and highest sensitivity were observed in the lever sign test and Lachman test in patients under anesthesia, respectively (meanly $41.9 \%$ and $98.9 \%$, respectively). The specificities of all the tests were excellent both under anesthesia and while awake.

To our knowledge, there was no study that investigated the importance of experience on these diagnostic tests simultaneously. To our knowledge, only one study demostrated that results of lever sign test were similiar when perfomed by undergraduate students and medical students compared to orthopaedic residents and fellows (Jarbo et al., 2017). Kilinc et al. (2016) reported that Lachman and anterior drawer tests perfectly correlate with KT-1000 in ACL reconstructed knees. The authors suggested using KT-1000 to determine the knee laxity if the examiner is inexperienced. However, our study revealed that the effect of experience on diagnostic tests was not significant, and knee laxity due to ACL rupture can be easily diagnosed even by an inexperienced examiner.

Interestingly, the interobserver reliabilities of these historical tests were not studied widely. Peeler et al. (2010) evaluated the interrater reliabilities of Lachman, anterior drawer, and pivot-shift tests in 112 patients, and the confirmation of ACL injury was made by surgery. The observers were divided into three groups: therapists, primary care physicians, and orthopedic surgeons. A coefficient of agreement $(\mathrm{Po})$ was calculated for the evaluation. Po values were; $0.45,0.57$, and 0.53 for Lachman, anterior drawer, and pivot-shift tests, respectively, which means a fair to moderate agreement. Yoon et al. (2014) used kappa statistics to evaluate interobserver reliabilities of the previous three tests. The conclusion was, $\kappa: 93, \kappa: 96$ and $\kappa: 87$ for Lachman, anterior drawer, and pivot-shift tests, respectively. Décary et al. (2016) concluded that the Lachman test was reliable in both low and high-quality studies in a recent meta-analysis. In another meta-analysis, Lange et al. (2015) could not conclude any of the tests to be reliable because of methodological quality flaws, different and partially inadequate reliability measures of included studies, and stated that further research is needed this area. Since the lever sign test is newer, the reliability study, including this test, is even more limited. Lichtenberg et al. (2018) evaluated four tests' interobserver reliabilities in 94 patients using the arthroscopic examination as a gold standard for ACL injury. There were two observers (an orthopedic surgeon and a physiotherapist). Cohen $\kappa$ was used for statistics. Interobserver reliabilities of lever sign, anterior drawer, Lachman and pivot-shift tests were $\kappa: 82, \kappa: 80, \kappa: 77$ and $\kappa: 84$, respectively. The examinations were performed while the patient was awake. Deveci et al. (2015) evaluated intraclass coefficient (ICC) values for determining the interobserver reliabilities of these four tests. The authors stated ICC values were; 0.89-0.96 for lever sign test, 0.85-0.91 for Lachman test, $0.82-0.88$ for pivot-shift test, and $0.84-0.93$ for anterior drawer test. The reason for high values in this study can be due to the limited number of observers $(\mathrm{n}=2)$. In our research, interobserver reliability of anterior drawer, pivot shift, and lever sign tests were found to be increased when the patients were under anesthesia compared with the awake condition ( 0.648 to $1,0.574$ to 0.863 , and 0.879 to 0.956 , respectively). 
Conversely, the Lachman test's interobserver reliability was decreased to -0.011 from 0.552 . We believe that eliminating the sensation of pain under anesthesia may have caused an increase in interobserver reliabilities. Still, it is difficult to explain the sharp decrease in intraobserver reliability of the Lachman test.

In the acute period after an ACL injury, factors such as; hemarthrosis and muscle spasms can alter the examiner's sense. In contrast, possible scarring of ruptured ACL to the posterior cruciate ligament can alter the chronic period's results. To our knowledge, there was no study simultaneously evaluated the interobserver reliabilities of four tests at both acute and chronic period of the injury. Our results showed that; interobserver reliabilities of the lever sign test (both awake and under anesthesia) and anterior drawer test (under anesthesia) were excellent at both acute and chronic periods. The Lachman test's interobserver reliability was poor both at acute and chronic periods when performed under anesthesia.

Mulligan et al. (2011) stated that Lachman and anterior drawer tests could be altered if the examiner's hand is small or if the patient's leg is large and muscular. That was particularly true. Therefore, we grouped the patients based on their BMI values. Interobserver reliabilities of both anterior drawer and Lachman tests in awake patients were lower in patients with a BMI value of $>25 \mathrm{~kg} / \mathrm{m}^{2}$, ( 0.658 to 0.566 and 0.641 to 0.199 , respectively). However, when the patients were under anesthesia, these reliabilities were not significantly affected. Lever sign test's interobserver reliability was excellent in both BMI groups when patients were either awake or under anesthesia. The lever sign test may be more reliable for detecting ACL rupture in overweight and obese patients by these findings.

This study has several limitations. Asymptomatic patients were not included in the study, which can make the strength of sensitivity and specificity evaluation lower. Sensitivities and specificities of the tests were not compared for the groups. Only the patients' data with a complete ACL injury were included in the assessment of interobserver reliability. Interobserver reliabilities could be altered if the patients with intact ACL were included in the study. Besides, MRI examination was not performed on contralateral knees, and these were considered intact ACL.

In conclusion, this study demonstrated that; the sensitivities of pivot shift and lever sign tests were low in both awake and anesthetized patients, while the specificities of all the tests were sufficient. These findings may mean that; pivot shift and lever sign tests are not adequate to predict the ACL rupture, but these tests help rule out the rupture. Overall, the anterior drawer test's interobserver reliability was perfect when the patient is examined under anesthesia. The lever sign test had the highest reliability in the awake condition in patients with a BMI of $>25 \mathrm{~kg} / \mathrm{m}^{2}$. Furthermore, the reliabilities of the anterior drawer, Lachman and pivot-shift tests in awake condition were found to be increased in patients with a chronic injury. Finally, the examiner's experience is not important for the physical diagnosis of ACL rupture.

\section{CONFLICT OF INTEREST}

No potential conflict of interest relevant to this article was reported.

\section{ACKNOWLEDGMENTS}

The authors received no financial support for this article.

\section{REFERENCES}

Abruscato K, Browning K, Deleandro D, Menard Q, Wilhelm M, Hassen A. Diagnostic accuracy of the lever sign in detecting anterior cruciate ligament tears: a systematic review and meta-analysis. Int J Sports Phys Ther 2019;14:2-13.

Décary S, Ouellet P, Vendittoli PA, Desmeules F. Reliability of physical examination tests for the diagnosis of knee disorders: evidence from a systematic review. Man Ther 2016;26:172-182.

Deveci A, Cankaya D, Yilmaz S, Özdemir G, Arslantaş E, Bozkurt M. The arthroscopical and radiological corelation of lever sign test for the diagnosis of anterior cruciate ligament rupture. Springerplus 2015;4:830.

Galway HR, Maclntosh DL. The lateral pivot shift: a symptom and sign of anterior cruciate ligament insufficiency. Clin Orthop Relat Res 1980; 147:45-50.

Jarbo KA, Hartigan DE, Scott KL, Patel KA, Chhabra A. Accuracy of the lever sign test in the diagnosis of anterior cruciate ligament injuries. Orthop J Sports Med 2017;5:2325967117729809.

Kilinc BE, Kara A, Celik H, Oc Y, Camur S. Evaluation of the accuracy of Lachman and Anterior Drawer Tests with KT1000 in the follow-up of anterior cruciate ligament surgery. J Exerc Rehabil 2016;12:363-367.

Lange T, Freiberg A, Dröge P, Lützner J, Schmitt J, Kopkow C. The reliability of physical examination tests for the diagnosis of anterior cruciate ligament rupture - A systematic review. Man Ther 2015;20:402411.

Lelli A, Di Turi RP, Spenciner DB, Domini M. The "lever Sign": a new clinical test for the diagnosis of anterior cruciate ligament rupture. Knee Surg Sports Traumatol Arthrosc 2016;24:2794-2797.

Lichtenberg MC, Koster CH, Teunissen LP, Oosterveld FG, Harmsen AM, 
Haverkamp D, Hoornenborg D, Berg RP, Bloemers FW, Faber IR. Does the lever sign test have added value for diagnosing anterior cruciate ligament ruptures? Orthop J Sports Med 2018;6:2325967118759631.

Logan MC, Williams A, Lavelle J, Gedroyc W, Freeman M. What really happens during the Lachman test? A dynamic MRI analysis of tibiofemoral motion. Am J Sports Med 2004;32:369-375.

Marshall JL, Wang JB, Furman W, Girgis FG, Warren R. The anterior drawer sign: what is it? J Sports Med 1975;3:152-158.

Mulligan EP, Anderson A, Watson S, Dimeff RJ. The diagnostic accuracy of the lever sign for detecting anterior cruciate ligament injury. Int J Sports Phys Ther 2017;12:1057-1067.

Mulligan EP, Harwell JL, Robertson WJ. Reliability and diagnostic accuracy of the Lachman test performed in a prone position. J Orthop Sports Phys Ther 2011;41:749-757.
Peeler J, Leiter J, MacDonald P. Accuracy and reliability of anterior cruciate ligament clinical examination in a multidisciplinary sports medicine setting. Clin J Sport Med 2010;20:80-85.

Reiman MP, Reiman CK, Décary S. Accuracy of the lever sign to diagnose anterior cruciate ligament tear: a systematic review with meta-analysis. Int J Sports Phys Ther 2018;13:774-788.

Scholten RJ, Opstelten W, van der Plas CG, Bijl D, Deville WL, Bouter LM. Accuracy of physical diagnostic tests for assessing ruptures of the anterior cruciate ligament: a meta-analysis. J Fam Pract 2003;52:689-694.

Torg JS, Conrad W, Kalen V. Clinical diagnosis of anterior cruciate ligament instability in the athlete. Am J Sports Med 1976;4:84-93.

Yoon KH, Lee SH, Park SY, Kang DG, Chung KY. Can physical examination predict the intraarticular tear pattern of the anterior cruciate ligament? Arch OrthopTrauma Surg 2014;134:1451-1457. 